CP, 2016, Vol.5 - No10, pp. 115-131. ISSN 2014-6752. Girona (Catalunya). Universitat de Girona. ORELLANA MÉNDEZ, O., BOSSIO SUAREZ, S., y CARHUALLANQUI CARHUAMACA, JhR.: Estilos comunicativos en estudiantes de Ciencias de la Comunicación de la Universidad Nacional del Centro del Perú. Recibido: 23/9/2016 - Aceptado: 17/10/2016

\title{
Estilos comunicativos en estudiantes de Ciencias de la Comunicación de la Universidad Nacional del Centro del Perú
}

\section{Communication styles in students of Sciences of the communication of the Universidad national of the center of the Peru}

\author{
Autores \\ Mg. Gaspar Orellana Méndez \\ Psicólogo y Magister en Psicología Educativa por la UNMSM, Docente principal en asignaturas de investi- \\ gación en la facultad de Ciencias de la Comunicación de la UNCP, \\ gaspar-50@hotmail.com \\ Mg. Sandro Bossio Suarez \\ Periodista, escritor y docente universitario, Magister en Tecnologías de la Comunicación mención en Educa- \\ ción Virtual por la Universidad Andina Simón Bolívar, \\ sbossios@yahoo.com \\ Lic. Jhony Raúl Carhuallanqui Carhuamaca \\ Comunicador por la Universidad Nacional del Centro del Perú y docente en la Facultad de Ciencias de la \\ Comunicación-Huancayo \\ jhony_by_01@hotmail.com
}

\section{RESUMEN}

La teoría clásica de los estilos de comunicación de Gudykunst et al (1994) considera que está conformada por la valoración del habla y la comunicación directa, y esta puede ser útil para explicar el comportamiento de los estudiantes universitarios a partir del estilo de comunicación que los caracterice. El estudio logra la validez y confiabilidad del Cuestionario de Estilos de Comunicación de Gudykunst et al (1994), establece baremos para el diagnóstico, caracteriza el estilo de comunicación y determinar la existencia de diferencias según semestre de estudio, en una muestra de 70 alumnos de la carrera profesional de Ciencias de la Comunicación de la Universidad Nacional del Centro del Perú (UNCP). En todo el estilo de comunicación, el $83.1 \%$ tiene puntajes de 24 a más, lo que señalaría que una gran mayoría valora el habla positivamente y prefiera la comunicación directa. Los dos tercios $(61,45 \%)$ de los sujetos valoran el habla y aprecian mucho la comunicación ya que presentan puntajes igual o mayores

\section{ABSTRACT}

The classical theory of communication styles Gudykunst et al (1994) considers that consists of the assessment of speech and direct communication, and this may be useful to explain the behavior of college students from the communication style that characterized. The study achieved the validity and reliability Style Questionnaire Communication Gudykunst et al (1994) provides scales for diagnosis, it characterizes the style of communication and determine the existence of differences according semester of study in a sample of 70 students the career of Communication Sciences of the National University of Central Peru (UNCP). Throughout the communication style, $83.1 \%$ have scores of 24 more, which would indicate that a large majority values the positive speech and prefers direct communication. Two-thirds $(61.45 \%)$ of the subjects valued greatly appreciate speech and communication as they have scores equal to or greater than 13. In direct communication, we note that $60.0 \%$ have scores of 14 
a 13. En la comunicación directa, observamos que el 60,0\% tiene puntajes de 14 a menos lo que indicarían que la mayoría de los examinados pertenecen a una cultura comunicacional de alta distancia jerárquica y colectivista, pero un $28,6 \%$ tienen puntajes iguales o mayores a 16 lo que indicaría que esta minoría tiene una cultura comunicacional de baja distancia jerárquica e individualista. El estilo hallado de elevada valoración del habla y preferencia por la comunicación directa (comunicación agresiva) correspondería a un estilo de liderazgo autoritario, lo cual explicaría la facilidad de llevarlos a acciones de medidas de fuerza a través de expresiones breves y directas, ajenas a que puedan ser corroboradas en su veracidad. Es necesario realizar estudios en muestras más grandes y a partir de los resultados obtenidos mejorar la formación académica de los estudiantes, organizando los mensajes en función del estilo de comunicación que los caracteriza.

PALABRAS CLAVES: Estilos de comunicación, validez, valoración del habla, comunicación directa, estudiantes universitarios.

\section{INTRODUCCIÓN}

El estudio de los estilos comunicativos de las personas en una institución es importante en la medida que dicho conocimiento nos permite adoptar medios acertados de interrelación para mejorar la convivencia y orientar a las personas al logro de objetivos institucionales.

Así, en la presente investigación, planteamos el proceso y los resultados de un estudio cuantitativo que determinará el estilo comunicativo de los alumnos de la facultad de Ciencias de la Comunicación de la UNCP que, en base a la diferenciación de sexo, ciclo de estudios y edad, nos mostrará los estilos comunicativos que están presentes y que deben tomarse en cada planificación u organización institucional, incluido el metodológico-educativo. unless what They indicate that most examinees belong to a communicational culture of hierarchical and collectivist high distance, but $28.6 \%$ had scores equal to or greater than 16 indicating that this minority has a communicational culture of low hierarchical and individualistic distance. The style found high valuation speech and preference for direct communication (aggressive communication) correspond to an authoritarian leadership style, which explains the ease of taking them to actions of force measures through short and direct expressions, beyond that they can be corroborated in its veracity. Studies are needed on larger samples and from the results obtained improve the academic training of students, organizing messages based communication style that characterizes.

KEYWORDS: Communication Styles, validit, assessment speaks, direct communication, college students

La Facultad de Ciencias de la Comunicación de la Universidad Nacional del Centro del Perú es una institución educativa de la región andina central del país que tiene alrededor de 310 alumnos de quienes desconocemos sus estilos comunicativos, variable importante en el proceso de interacción y del aprendizaje, pues conocerlas nos permitiría un mejor manejo. A lo largo de su historia la Facultad ha pasado por experiencias de acciones tanto de docentes y estudiantes, en las que a través del rumor y mensajes sencillos y directos han movilizado a estos estamentos en la consecución de sus demandas de gestión o académicas. El año 2014, para que la administración de la radio universitaria pase a la facultad, con expresiones de frases breves y directas se movilizo a estudiantes para que tomen medidas de fuerza y se lograra su administración, cuando el problema de fondo era la 
burocracia y dejadez de autoridades de la universidad y facultad, quienes no tomaron en cuenta la lectura de resoluciones emanadas por el Consejo Universitario, en la que se señalaba los plazos y procedimientos para transferir la radio universitaria y que la facultad no había cumplido, que de haberse hecho no habría razón para tomar esas medidas de fuerza.

Por otro lado en nuestro medio solo contamos con un instrumento propio para medir los estilos comunicativos, el Cuestionario de Estilo de Comunicación de Ludeña (2007).

En otros contextos existen instrumentos de medición de los estilos comunicativos como el Inventario de Estilos de Comunicación de Sánchez-Aragón y Díaz-Loving (2003), el Cuestionario de Perfil de Conductas Comunicativas del Empleado Superior (CPCCES) de Varona (2007), la Escala de Estilos de Comunicación y Satisfacción Sexual de Alvarado, García y Ramírez (2011), el Cuestionario Estilos de Mensajes en el Manejo del Conflicto (CMMS) de Luna, Laca y Cedillo (2012), la Escala de Comunicación Padres-Adolescentes (PACS) de Barmes y Olson (1982) adaptado para argentina por Schmidt, Messoulam, Molina y Abal (2008), y el Cuestionario de Estilo de Comunicación de Alto y Bajo Contexto (62 ítems) de Gudykunst, Ting-Toomey, Sudweeks y Stewart (1996).

En este estudio se hace necesario no solo adaptar y validar el Cuestionario de Estilos Comunicativos (8 ítems) de Gudykunst, Matsumoto, Ting-Toomey, Nishida, Kim y Heyman (1994) y establecer baremos, sino también caracterizar el tipo y nivel de estilos comunicativos y determinar la existencia de diferencias significativas en los estilos comunicativos según sexo, ciclo de estudios y edad en los alumnos de la facultad de Ciencias de la Comunicación de la UNCP.

\subsection{La teoría}

En el plano teórico, existen diferentes puntos de vista entorno a los estilos de comu- nicación, planteando diferentes números de componentes de esta variable, tal como MiIler, Nunnally y Wackman (1976), describen cuatro estilos de comunicación verbal, el primero, sociable, amigable, juguetón, optimista; el segundo, demandante, persuasivo, competitivo, controlador, directivo, ofensivo y prejuicioso; el tercero, elabora, investiga, desea y es propositivo y; el cuarto, comunicador, comprensivo y cooperativo. Satir (1986), plantea seis estilos de comunicación: "Aplacar", "Culpar", "Supra-razonar", "Distraer", "Irrelevante" y "Apertura". Van-der Hofstadt (2003), plantea un estilo agresivo, estilo pasivo y un estilo asertivo. Chamoun-Nicolas (s/f), en referencia a Morganthau, dice que los estilos de comunicación serían: Relacionador, Persuasivo, Analítico y Directivo. Finalmente, Fensterheim y Baer (2003) refieren la existencia de tres estilos básicos de comunicación en base a la actitud hacía el interlocutor: Pasividad o no-asertividad, agresividad y asertividad.

En el estudio, asumimos el punto de vista de Gudykunst, et al (1994) y Gudykunst, et al (1996), quienes indican la existencia de dos estilos generales de comunicación verbal estilo directo-indirecto en cuanto a la revelación de intenciones y estilo sucinto-exacto-exagerado, en cuanto a la calidad del habla que se valora. Esta es una aproximación conceptual a los estilos comunicativos que se resume con la definición de un conjunto de atributos y valores que cualquier individuo posee: la valoración del habla y la comunicación directa.

Por otro lado, en relación a los estilos de comunicación de Alto y Bajo Contexto, tanto Gudykunst, et al (1996), como Hall (1978) consideran que la comunicación de Bajo Contexto involucra el uso de mensajes explícitos y directos en los cuales el significado está contenido principalmente en los mensajes transmitidos (este comunicador es abierto, auto-divulgador, los silencios significan que no hay nada más que decir, se es preciso), la comunicación de Alto 
Contexto involucra el uso de mensajes implícitos e indirectos, en los cuales los significados están dentro de la persona o en el contexto socio-cultural (el comunicador no es abierto, su silencio comunica más que su voz, son sensibles a los otros). Así, la gente de una cultura puede usar ambos tipos de comunicación, pero uno tiende a ser predominante.

Estas dos dimensiones culturales de individualismo-colectivismo de Gudykunst, et al (1996) y los estilos comunicativos de Hall (1978), mantienen una fuerte relación. Las formas de comunicación explicita, son más propias del síndrome cultural del individualismo, mientras que la comunicación implícita, es utilizada por individuos de culturas colectivistas.

Además señala Hall (1978), que las culturas de comunicación implícita son, de mayor a menor, las asiáticas (China, Corea, Japón), las árabes y del mediterráneo oriental (Turquía), las de América Latina y las de Europa del Sur (Grecia, España e Italia).Las culturas inglesa y francesa son mixtas. Las de bajo contexto o estilo explicito son Estados Unidos, la escandinava y la del área germánica, siendo suiza Germánica, la cultura de menor énfasis en el contexto.

Los autores mencionan también un estilo deferente y ritual, en cuanto a las relaciones, son simétricas o asimétricas, el estilo directo permite percibir al emisor como de mayor status y muestra mayor intimidad. La mayor cortesía se asocia con el menor estatus del hablante y la no directa crea una impresión de menor status y cercanía. El uso de lenguaje jerárquico es utilizado principalmente en Asia y Latinoamérica.

En situaciones de incertidumbre las culturas de baja evitación a la incertidumbre toleran mejor la ambigüedad, integran mejor las viejas y nuevas ideas y cambian sus creencias de forma acorde y tienen mejor disposición a buscar información sobre los extraños para establecer una comunicación efectiva. En las culturas de baja tolerancia a la ambigüedad se promueven estructuras cerradas y previsibles, intentan mantener sus creencias y rechazar las ideas que son diferentes.

También se señala el uso del silencio. En las sociedades colectivistas, de alto contexto y comunicación implícita, la comunicación efectiva requiere de la habilidad para interpretar pausas en la conversación, el silencio sería interpretado como acuerdo. Mientras que en las individualistas (comunicación explicita), no hace mucho uso del silencio y si lo utiliza sería una manifestación de manipulación, falta de intención o iniciativa.

Después, se encontró que en las sociedades individualistas existe mayor expresión verbal de las emociones, hablar con franqueza es más valorado, contrariamente en las colectivistas hay menor expresión verbal y elaboración cognitiva.

En el colectivismo las emociones son consideradas de poca importancia por lo que la ausencia de expresión verbal es irrelevante, en sociedades como China la acción se pone de relieve, en la interacción y comunicación, en detrimento de la expresión verbal. Donde es menos importante conocer historia, sentimientos personales ya que el estatus, afiliación y reglas contextuales son las que determinan el fin y sentido de la interacción. En el individualismo hablar y compartir las emociones es importante porque permite decidir cuál es el grado de intimidad y confianza que se va establecer en una relación.

Si bien los estudios señalan que Latinoamérica, y dentro de ella el Perú, son sociedades indicadas por estudios como colectivistas y de comunicación indirecta, el caso de estudiantes de comunicaciones sería una excepción, debido a que dicha carrera, a diferencia de otras, demanda y promueve el desarrollo de características comunicativas más eficaces como el lenguaje explícito, el énfasis en la conversación parar exponer claramente su punto de vista con el fin de 
informar y convencer a su interlocutor. Entonces, con base a la teoría del estilo de comunicación de Gudykunst, et al (1994), suponemos que los participantes utilizarían predominantemente el estilo comunicativo directo, y su valoración del habla seria alto, prioritariamente del tipo exagerado, ya que, este último requiere riqueza, expresividad verbal, repetición, lo que facilita la comunicación entre hablante y receptor, satisface las necesidades del profesional de la comunicación, además es típico del colectivismo latino y mediterráneo.

\subsection{Los antecedentes}

Varona (2007) investigó las diferencias en el estilo de comunicación entre empleados finlandeses y mexicanos cuando se comunican con sus jefes. Basado en la definición de Norton (1983) sobre Estilos de Comunicación ("es la manera como se comunican entre sí verbal y no verbalmente") utilizó el Cuestionario de Perfil de Conductas Comunicativas del Empleado Superior (CPCCES), compuesto por 68 preguntas que analiza 14 dimensiones en una escala tipo Likert. La muestra fue recogida en cada país: tres empresas Finlandesas y en México una Universidad privada. Concluye que en general los finlandeses escuchan más a sus jefes, así mismo, los mexicanos emplean más la comunicación verbal y no verbal, como también la comunicación formal y no formal. En las comunicaciones los mexicanos aluden más temas vinculados a la política o religión, en cambio los finlandeses más sobre deportes o recreación. Los empleados mexicanos esperan recibir comentarios positivos y muestran mayor apertura comunicativa por ello usan más los canales de comunicación.

Armenta y Díaz-Loving (2008), estudiaron la comunicación y satisfacción en la Interacción de pareja, aplicaron el Inventario de Estilos de Comunicación de Sánchez-Aragón y Díaz-Loving (2003) versión corta de Roca (2003), a 114 parejas heterosexuales, mayores de 18 años, que viven juntas alrededor de seis años. Concluyeron que cuan- do uno de ellos no se comunica de forma destructiva, fría, intolerante, severa, inexpresiva o distante, el otro es más platicador, oportuno y optimista al comunicarse, lo que produce una relación armoniosa.

Alvarado, García y Ramírez (2011) estudiaron la relación entre estilos de comunicación y satisfacción sexual en estudiantes universitarios, 278 hombres y mujeres de nueve carreras profesionales, con un rango de edad de 20 a 30 años. Recogieron datos con la Escala de Estilos de Comunicación y Satisfacción Sexual auto elaborada y que tiene 61 reactivos. Concluyen que el uso de un determinado estilo de comunicación entre los miembros de la pareja, influye directamente en su satisfacción sexual.

Ludeña (2007), investiga los estilos de comunicación que establece la enfermera según opinión de los padres de niños quemados del Instituto Nacional de Salud del Niño (INSN). Utilizó una Lista de Cotejo y un cuestionario con 31 reactivos que analiza dos dimensiones: Comunicación verbal y la comunicación no verbal. La muestra estuvo conformada por 41 padres de familia que voluntariamente accedieron al estudio y se encontraban en la sección de quemados en noviembre de 2006. Concluyó que la opinión que tienen los padres de los niños quemados hacia el estilo de comunicación verbal de la enfermera es de medianamente favorable a favorable, en cambio, cuando nos referimos al estilo de comunicación no verbal, el resultado es de medianamente favorable a desfavorable.

Flores (2010), estudió el tipo de liderazgo del director y los estilos de comunicación de los docentes, estudiantes y personal administrativo del Instituto Superior Pedagógico Público de Ayacucho. El trabajo se desarrolla con 195 personas entre docentes, administrativos, los datos se recogieron con un cuestionario ad hoc de 30 preguntas. Concluyeron que existe una relación significativa entre el tipo de liderazgo liberal del director y el estilo de comunicación pa- 
siva, entre el tipo de liderazgo autoritario del director y el estilo de comunicación agresiva y entre el tipo de liderazgo democrático del director y el estilo de comunicación asertivo.

Luna, Laca y Cedillo (2012), analizan correlaciones significativas entre patrones de toma de decisiones, autoconfianza como tomador de determinaciones, patrones de comunicación familiar y estilos de mensajes en el manejo de conflictos con los padres en 412 adolescentes bachilleres con edades de entre 15 y 19 años, quienes respondieron una versión adaptada del Cuestionario de Estilos de Mensajes en el Manejo del Conflicto, el Cuestionario Melbourne de Toma de Decisiones y la Escala de Autoconfianza como Tomador de Decisiones, así como la Escala de Comunicación Padres-Adolescentes. Entre los resultados, se encontraron correlaciones significativas entre comunicación familiar abierta, estilos de afrontamiento del conflicto orientados hacia la cooperación, y autoconfianza y vigilancia en la toma de decisiones. Se discuten estos $\mathrm{y}$ otros resultados relevantes.

Sánchez y Díaz (2003), estudiaron a los patrones y estilos de comunicación presentes durante el intercambio simbólico llevado a cabo por las parejas. En este trabajo particular, se identificaron los estilos utilizados por parejas mexicanas mediante metodologías etnopsicológicas y en base a las tipologías encontradas en la literatura (p.e. Satir, 1986; Miller, Nunnally y Wackman, 1976), con el fin de desarrollar un instrumento de medida de los estilos de comunicación universal pero culturalmente sensible. Los resultados indican dimensiones válidas, confiables y culturalmente sensibles en la medición de la gama de estilos positivos y negativos que en México las personas con pareja usan para comunicarse, surgiendo estilos característicos como: Empático y Social Expresivo, Social Afiliativo, Social Auto-modificador, Simpático y Social Normativo, Maquiavélico, Chismoso e Hiriente e Irritante Expresivo.
Schmidt, et al (2008), adaptaron la Escala de Comunicación Padres-Adolescentes de Barnes y Olson, (1982), revisaron los ítems por expertos (de 35 a 26 ítems) y aplicaron a 591 adolescentes varones (38\%) y mujeres (62\%) de 16 años de Buenos Aires. Se otorgó validez y confiabilidad al instrumento y el Análisis factorial rotación Varimax, determino una escala de 5 variaciones con 22 ítems y tres factores: Comunicación Abierta con 12 ítems (positiva), Problemas en la Comunicación con 7 ítems (negativa) y Restricción-Evitación con 3 ítems.

Prior, Manzano, Villar, Caparrós, Juan y Luz (2011), abordan la relación entre los estilos comunicativos de los estudiantes universitarios, su vinculación en la universidad y el nivel de adaptación psicosocial. Se analizan distintos estilos comunicativos a través de la Escala de Estilos Comunicativos (CSM) de Norton (1978) aplicada a 529 estudiantes universitarios.

Los resultados indican la existencia de diferencias de género con respecto a algunos patrones comunicativos pero no en relación con la vinculación universitaria. Se constata también una relación estadísticamente significativa, aunque no muy elevada, entre los estilos comunicativos y la capacidad de los estudiantes para vincularse en el contexto universitario. Tanto los estilos comunicativos como la vinculación universitaria contribuyen a la explicación de la sintomatología afectiva, pero sólo los estilos comunicativos polémico y amigable contribuyen a la explicación del consumo de sustancias.

\section{MÉTODO}

El tipo de estudio es descriptivo transversal, porque se recoge datos en un solo momento y busca describir los estilos comunicativos, así como determinar diferencias según género, edad y semestre de estudio de la carrera profesional de Ciencias de la Comunicación de la UNCP. Se utilizan el diseño descriptivo simple para caracterizar la variable de estudio, y el descriptivo comparativo para determinar si existen diferencias 
en los estilos comunicativos según variables sociodemográficas.

\subsection{Participantes}

Por su lado, la muestra la conforman 70 alumnos, 28 varones (40\%) y 42 mujeres $(60 \%)$, de la carrera profesional Ciencias de la Comunicación de la Universidad Nacional del Centro del Perú, seleccionados según la técnica aleatoria estratificada. Las edades van de 17 a 24 años y su promedio es de 19,74 y su D.T de 5,17. Participaron alumnos de los diez semestres académicos.

\section{2. Instrumento}

En cuanto al instrumento, se aplicó el cuestionario de Estilos de Comunicación de Gudykunst, et al (1994). Consta de dos categorías: valoración del habla, con los ítems $1,2,3$ y 4 y comunicación directa al que le correspondían las preguntas $5,6,7$ y 8 .En una escala tipo Likert cada sujeto marca su alternativa de 1 a 5 que va de nunca a siempre. Las respuestas se califican de 1 a 5 .

Si las puntuaciones son altas se considera que valoran más el hablar y la comunicación directa. En valoración del habla, puntuaciones de 13 o más indica que se aprecia mucho la comunicación y cuando es 11 o menos el estilo de comunicación es implícito. En comunicación directa, de 16 o más señala la pertenencia a una cultura de baja distancia jerárquica e individualista, mientras que si la puntuación es 14 o menos, advierte una alta distancia jerárquica y colectivista.

En el estudio se halló la validez empírica ítem test a través de la r de Pearson Corregida, cuyos valores fueron de .267 a .563. En la confiabilidad el Alfa de Cronbach de .714 indica estabilidad de las puntuaciones.

\section{3. Procedimientos}

A una muestra aleatoria de 74 alumnos de la carrera de Ciencias Comunicación que cursaban el periodo lectivo 2013-II se aplicó el Cuestionario de Estilos de Comunicación de Gudykunst, et al (1994), en sus aulas de manera individual y de pequeños grupos.
Una vez revisado los cuestionarios aplicados nos quedamos con una muestra valida de 70 participantes, y con la base de datos en el programa estadístico SPSS versión 15, se procesaron los estadísticos correspondientes y se elaboraron las tablas y figuras que permitieron dar respuesta a los problemas planteados.

A fin de lograr la validez y confiabilidad del instrumento se aplicó la $r$ de Pearson Corregida y el Alfa de Cronbach, respectivamente. Para la caracterización de los estilos comunicativos de los alumnos de la UNCP se usó el estadístico media aritmética y la desviación estándar. Para determinar las diferencias de los estilos comunicativos según sexo, edad y ciclo de estudio se aplicó la t de student y el anova al 0.05 de significación. Para determinar la escala se sumó y resto una desviación estándar al puntaje promedio para hallar así tres niveles.

\section{RESULTADOS}

\subsection{Validez y confiabilidad del cuestiona-} rio de Estilos Comunicativos

El alfa de Cronbach obtenido fue de 0.714, valor aceptado para considerarse que las puntuaciones presenten estabilidad. La tabla uno presenta estadísticos de la $r$ de Pearson Corregida y vemos que el análisis de validez empírica ítem test arroja valores iguales o mayores de 0.20 en todos los ítems, que van desde .267 en el ítem uno (Me gusta mucho hablar y compartir en situaciones sociales) hasta .532 en el ítem seis (Voy directo al grano cuando hablo con las personas). Por lo visto resultan validos los ocho ítems. 
Tabla 1: Media, D.T y r de Pearson Corregida de los ítems de los estilos de comunicación ( $n=70)$.

\section{Ítems Estilos Comunicativos}

1. Me gusta mucho hablar y compartir en situaciones sociales.

2. Pienso que las personas que hablan poco son aburridas

y poco interesantes.

3. No me gustan las conversaciones superficiales,

ni banales (hablar de la moda, futbol, etc.).

4. Cuando otras personas manifiestan una opinión contraria

a la mía, o estoy en desacuerdo con ellas, lo manifiesto

abiertamente y en forma emocional.

5. Espero que los otros entiendan o deduzcan mi opinión

sin que tenga que decirla directamente.

6 . Voy directo al grano cuando hablo con las personas.

7. Uso expresiones que matizan lo que digo cuando hablo

(por ejemplo "puede ser", "quizás").

8. Le presto más atención a lo que las personas

dicen directamente, y no a lo que intentan decir o no dicen.
Media D.T. $\quad$ de P Corregida

$3.64 \quad .917 \quad .267$

$\begin{array}{lll}3.10 & 1.144 & .404\end{array}$

$\begin{array}{lll}3.27 & 1.128 & .419\end{array}$

$\begin{array}{lll}3.57 & 1.071 \quad .505\end{array}$

$\begin{array}{lll}3.39 & 1.054 & .309\end{array}$

$\begin{array}{lll}3.81 & .967 & .532\end{array}$

$\begin{array}{lll}3.56 \quad .862 & .467\end{array}$

$\begin{array}{lll}3.61 & .952 \quad .354\end{array}$

\subsection{Caracterización del estilo de comu- nicación}

También la tabla uno presenta los puntajes promedios y la desviación típica, de una puntuación de 1 a 5 , la media más alta se dio en el ítem 6: Voy directo al grano cuando hablo con las personas y la más baja en el ítem 2: Pienso que las personas que hablan poco son aburridas y poco interesantes, el resto de reactivos obtuvieron medias por encima del puntaje medio de 3 evidenciado niveles altos. La mayor dispersión de los datos se dio en el ítem 2: Pienso que las personas que hablan poco son aburridas $y$ poco interesantes y la menor dispersión en el ítem 7: Uso expresiones que matizan lo que digo cuando hablo (por ejemplo "puede ser", "quizás"). , la mayoría de ítems presentan dispersión moderada.

La tabla dos, presenta la media y desviación estándar de los estilos comunicativos y sus dimensiones. De una puntuación mínima a

obtener de 8 y una máxima de 40 , con un punto central arbitrario de 24, se obtuvo la media de 27.96 en la variable estilos comunicativos lo que indicaría un nivel ligeramente superior al punto medio. En cada una de las dos dimensiones se puede obtener un puntaje mínimo de 4 y máximo de 20 y el punto medio arbitrario seria de 12 , en la valoración del habla el puntaje promedio es de 13.59 y en la comunicación directa es de 14.37 lo cual nos indica un nivel ligeramente por encima del promedio, siendo la más alta en la comunicación directa. La dispersión de los datos de los estilos comunicativos es moderada (DT=4.69), la más baja dispersión lo tiene la dimensión Comunicación directa $(D T=2.51)$ y la más alta la valoración del habla (DT=2.76). En tal sentido las dimensiones y toda la variable se encontrarían en el nivel ligeramente alto, y sus datos son moderadamente dispersos.

Tabla 2: Media y D.T. de los estilos de comunicación y sus dimensiones $(n=70)$

Estadísticos

Media

Desv. Típica

Puntaje Mínimo

Puntaje Máximo

\section{Valoración del Habla}

13.59
2.764
8
24

24

\section{Comunicación Directa Estilo Comunicativo}

$\begin{array}{ll}14.37 & 27.96 \\ 2.509 & 4.692 \\ 9 & 19 \\ 20 & 40\end{array}$




\subsection{Descripción de los estilos de comu- nicación}

La tabla tres presenta los datos descriptivos de frecuencias y porcentajes de cada uno de los ítems del cuestionario de estilos de comunicación que perciben los alumnos de Ciencias de la Comunicación, según ítems y alternativas de respuestas.

Tabla 3: Frecuencias y porcentaje de los ítems del Estilo Comunicativo, según escala.

\begin{tabular}{lllll} 
Ítems Estilos Comunicativos & \multicolumn{2}{c}{ Nunca } & \multicolumn{2}{c}{ Casi Nunca } \\
& $f$ & $\%$ & $f$ & $\%$ \\
1. Me gusta mucho hablar y ... & 2 & 2.9 & 4 & 5.7 \\
2. Pienso que las personas ... & 5 & 7.1 & 17 & 24.3 \\
3 No me gustan las convers... & 4 & 5.7 & 13 & 18.6 \\
4. Cuando otras personas ... & 2 & 2.9 & 10 & 14.3 \\
5. Espero que los otros entie... & 2 & 2.9 & 15 & 21.4 \\
6. Voy directo al grano cuan... & 2 & 2.9 & 6 & 8.6 \\
7. Uso expresiones que ma... & 0 & 0.0 & 7 & 10.0 \\
8. Le presto más atención a ... & 1 & 1.4 & 9 & 12.9
\end{tabular}

Los resultados de la tabla tres demuestran lo siguiente:

Que el $60,0 \%$ de los alumnos señalan que siempre y casi siempre gustan de hablar y compartir en situaciones sociales y que nos indica que la mayoría de los estudiantes valora positivamente el habla, en tanto que el $8,6 \%$ no lo valora y el $31,45 \%$ lo valora moderadamente.

Que el 34,3\% de los alumnos señalan que siempre y casi siempre piensan que las personas que hablan poco son aburridas y poco interesantes y que nos indica que un tercio de los estudiantes valora positivamente el habla, en tanto que los otros tercios lo valoran moderadamente (34.3\%) o no lo valoran $(31,4 \%)$.

Que el $40.0 \%$ de los alumnos señalan que siempre y casi siempre no le gustan las conversaciones superficiales, ni banales (hablar de la moda, futbol, etc.) y que nos indica que la mayoría de los estudiantes valora positivamente el habla, en tanto que el $24.3 \%$ no lo valora y el $35.7 \%$ lo valora moderadamente.

Que el $55.7 \%$ de los alumnos señalan que siempre y casi siempre que cuando otras personas manifiestan una opinión contraria o está en desacuerdo con ellas, lo manifiesta abiertamente y en forma emocional, lo

\begin{tabular}{llllll}
\multicolumn{2}{c}{ A Veces } & \multicolumn{2}{c}{ Casi Siempre } & \multicolumn{2}{c}{ Siempre } \\
$\mathrm{f}$ & $\%$ & $\mathrm{f}$ & $\%$ & $\mathrm{f}$ & $\%$ \\
22 & 31.4 & 31 & 44.3 & 11 & 15.7 \\
24 & 34.3 & 14 & 20.0 & 10 & 14.3 \\
25 & 35.7 & 16 & 22.9 & 12 & 17.1 \\
19 & 27.1 & 24 & 34.3 & 15 & 21.4 \\
16 & 22.9 & 28 & 40.0 & 9 & 12.9 \\
10 & 14.3 & 37 & 52.9 & 15 & 21.4 \\
27 & 38.6 & 26 & 37.1 & 10 & 14.3 \\
17 & 24.3 & 32 & 45.7 & 11 & 15.7
\end{tabular}

que nos indica que la mayoría de los estudiantes valora positivamente el habla, en tanto que el $17.2 \%$ no lo valora y el $27.1 \%$ lo valora moderadamente.

Que el $53.8 \%$ de los alumnos señalan que siempre y casi siempre esperan que los otros entiendan o deduzcan su opinión sin que tengan que decírsela directamente y que nos indica que la mayoría de los estudiantes valora negativamente la comunicación directa, en tanto que el $24.3 \%$ lo valora y el $22.9 \%$ lo valora moderadamente.

Que el $74.3 \%$ de los alumnos señalan que siempre y casi siempre van directo al grano cuando hablan con las personas y que nos indica que la mayoría de los estudiantes valora positivamente la comunicación directa, en tanto que el $11.4 \%$ no lo valora y el $14.3 \%$ lo valora moderadamente.

Que el $51.4 \%$ de los alumnos señalan que siempre y casi siempre usan expresiones que matizan lo que dicen cuando habla (por ejemplo" puede ser", "quizás") y que nos indica que la mayoría de los estudiantes no valora positivamente la comunicación directa, en tanto que el $10.0 \%$ si lo valora y el $38.6 \%$ lo valora moderadamente.

Que el $61.4 \%$ de los alumnos señalan que siempre y casi siempre prestan más aten- 
ción a lo que las personas dicen directamente, y no a lo que intentan decir o no dicen y que nos indica que la mayoría de los estudiantes valora positivamente la comunicación directa, en tanto que el $14.3 \%$ no lo valora y el $24.3 \%$ lo valora moderadamente.
A continuación se presentan los datos que describen los estilos de comunicación y sus dimensiones de los alumnos de Ciencias de la Comunicación de la UNCP, a través de tablas de frecuencias y porcentajes.

Tabla 4: Frecuencias y porcentaje de los puntajes, según dimensiones de los estilos comunicativos

\begin{tabular}{llcl} 
Puntajes & \multicolumn{3}{c}{ Valoración del Habla } \\
& $\mathbf{f}$ & $\%$ & \% Acum. \\
8 & 1 & 1.4 & 1.4 \\
9 & 2 & 2.9 & 4.3 \\
10 & 5 & 7.1 & 11.4 \\
11 & 8 & 11.4 & 22.9 \\
12 & 11 & 15.7 & 38.6 \\
13 & 10 & 14.3 & 52.9 \\
14 & 13 & 18.6 & 71.4 \\
15 & 3 & 4.3 & 75.7 \\
16 & 5 & 7.1 & 82.9 \\
17 & 4 & 5.7 & 88.6 \\
18 & 5 & 7.1 & 95.7 \\
19 & 9 & 0.0 & 95.7 \\
20 & 3 & 4.3 & 100.0
\end{tabular}

La tabla cuatro, presentan la distribución de frecuencias y los porcentajes de las dimensiones Valoración del Habla y Comunicación Directa. En el primer caso observamos que el $22,9 \%$ tiene puntajes de 11 a menos lo que indicarían que casi un tercio de los sujetos no valoran el habla siendo su comunicación implícita, en tanto los dos tercios restantes valoran positivamente el habla ya que presentan puntajes igual o mayores a $13(61,45 \%)$. En esta misma tabla, en la dimensión comunicación directa, observamos que el $60,0 \%$ tiene puntajes de 14 a menos lo que indicarían que la mayoría de los examinados pertenecen a una cultura comunicacional de alta distancia jerárquica y colectivista, pero un $28,6 \%$ tienen puntajes iguales o mayores a 16 lo que indicaría que esta minoría tiene una cultura comunicacional de baja distancia jerárquica e individualista.

La tabla cinco, presentan la distribución de frecuencias y los porcentajes de los estilos de comunicación de estudiantes universitarios de la carrera profesional de Ciencias de la Comunicación. De una puntuación de 8 a

\begin{tabular}{lcl}
\multicolumn{3}{l}{ Comunicación Directa } \\
$\mathbf{f}$ & $\%$ & $\%$ Acum. \\
0 & 0.0 & 0.0 \\
1 & 1.4 & 1.4 \\
2 & 2.9 & 4.3 \\
3 & 4.3 & 8.6 \\
10 & 14.3 & 22.9 \\
11 & 15.7 & 38.6 \\
15 & 21.4 & 60.0 \\
8 & 11.4 & 71.4 \\
8 & 11.4 & 82.9 \\
3 & 4.3 & 87.1 \\
3 & 4.3 & 91.4 \\
2 & 2.9 & 94.3 \\
4 & 5.7 & 100.0
\end{tabular}

40 el punto medio arbitrario es de 24 y observamos que el $83.1 \%$ tiene puntajes de 24 a más, lo que indicaría que una gran mayoría valora el habla positivamente y prefiera la comunicación directa.

Tabla 5: Frecuencias y porcentaje de las puntuaciones de la variable Estilos Comunicativos

$\begin{array}{cccc}\text { Puntajes } & \text { Frecuencia } & \text { Porcentaje } & \begin{array}{c}\text { Porcentaje } \\ \text { acumulado }\end{array} \\ 19 & 1 & 1.4 & 1.4 \\ 20 & 1 & 1.4 & 2.9 \\ 21 & 2 & 2.9 & 5.7 \\ 22 & 2 & 2.9 & 8.6 \\ 23 & 6 & 8.6 & 17.1 \\ 24 & 2 & 2.9 & 20.0 \\ 25 & 7 & 10.0 & 30.0 \\ 26 & 9 & 12.9 & 42.9 \\ 27 & 7 & 10.0 & 52.9 \\ 28 & 10 & 14.3 & 67.1 \\ 29 & 2 & 2.9 & 70.0 \\ 30 & 4 & 5.7 & 75.7 \\ 31 & 3 & 4.3 & 80.0 \\ 32 & 2 & 2.9 & 82.9 \\ 33 & 2 & 2.9 & 85.7 \\ 34 & 2 & 2.9 & 88.6 \\ 35 & 1 & 1.4 & 90.0 \\ 37 & 4 & 5.7 & 95.7 \\ 38 & 2 & 2.9 & 98.6 \\ 40 & 1 & 1.4 & 100.0\end{array}$




\subsection{Diferencias de los Estilos de Comuni- cación según variables demográficas}

En esta parte se presentan los datos hallados en tablas, para determinar si hay diferencias significativas en los estilos comunicativos y sus dimensiones, según variables de sexo, edad y semestre de estudio en los alumnos de la carrera profesional de Ciencias de la Comunicación de la UNCP, a través de la media, desviación estándar, la prueba $t$ de student y el anova. En la tabla seis se comprueba que no existen dife- rencias significativas en los estilos comunicativos, ni en las dimensiones valoración del habla y comunicación directa, según la variable sexo, pese a que las mujeres obtienen puntajes promedios más superiores. Los valores en la prueba $t$ al comparar los puntajes promedios no son significativas al 0,05 . En síntesis los alumnos varones y mujeres no difieren en la percepción que tienen de los estilos comunicativos y sus dimensiones.

Tabla 6: Diferencias de los Estilos de Comunicación y sus dimensiones, según sexo.

$\begin{array}{lllllll}\text { Variables } & \text { SEXO } & \mathbf{N} & \text { Media } & \text { D.T. } & \mathbf{t}(\mathbf{p}) \\ \text { Valoración del Habla } & \text { varón } & 28 & 12.86 & 2.563 & -1.831(.071) \mathrm{ns} \\ & \text { mujer } & 42 & 14.07 & 2.815 & \\ \text { Comunicación Directa } & \text { varón } & 28 & 13.93 & 2.107 & -1.210(.231) \mathrm{ns} \\ & \text { mujer } & 42 & 14.67 & 2.729 & & \\ & & & & & & \\ \text { Estilos Comunicativos } & \text { varón } & 28 & 26.79 & 3.755 & -1.730 & (.088) \mathrm{ns} \\ & \text { mujer } & 42 & 28.74 & 5.118 & \end{array}$

ns no significativo

La Tabla siete nos presenta los valores para determinar diferencias significativas en los estilos comunicativos y sus dimensiones, según la variable edades de 17 a 23 años. Los valores de la ANOVA hallados y su nivel de significación demuestran que no existen diferencias significativas entre alumnos de 17 a 23 años de edad en la variable de estu- dio y sus dimensiones en universitarios de Ciencias de la Comunicación de la UNCP, notándose que los de 19 años presentan las puntuaciones más altas en los estilos comunicacionales, la valoración del habla y la comunicación directa. 
Tabla 7: Diferencias de los estilos de comunicación y sus dimensiones, según edad

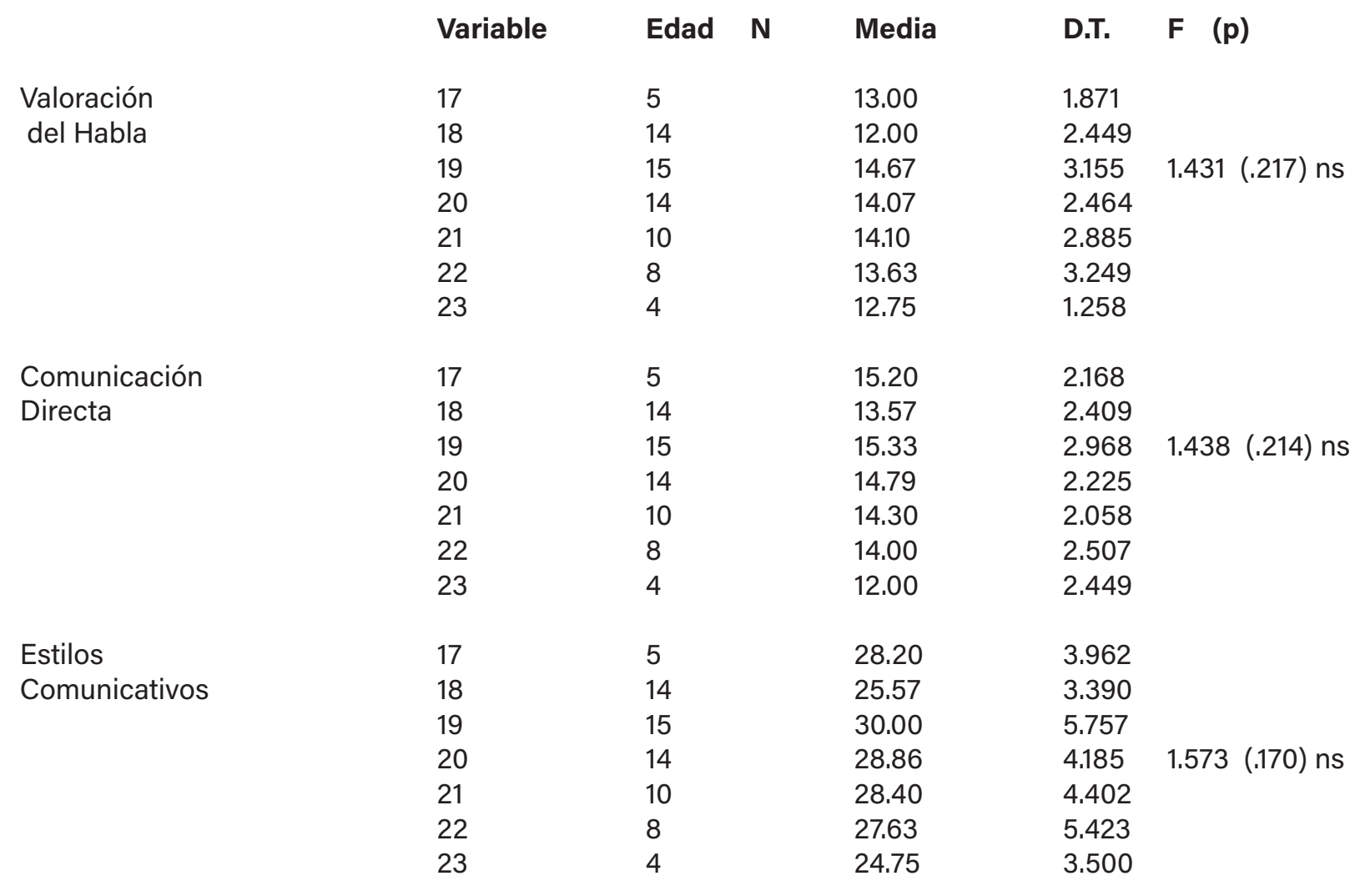

ns :no significativo

En la tabla ocho se demuestra que si existen diferencias significativas en los estilos comunicativos $(F=8.007$ y $p=.000)$, valoración del habla $(F=4.946$ y $p=.000)$ y comunicación directa $(F=7.044$ y $p=.000))$, según la variable semestre de estudio, donde los de octavo y noveno semestre obtienen puntajes promedios más superiores. Los alumnos de Ciencias de la Comunicación difieren significativamente en la percepción que tienen de los estilos comunicativos y sus dimensiones, según semestre de estudio.

Tabla 8: Diferencia de los estilos de comunicación y sus dimensiones, según semestre de estudio

$\begin{array}{llllll}\text { Variable } & \text { Semestre } & \text { N } & \text { Media } & \text { D.T. } & \text { F (p) } \\ \text { Valoración } & \text { Primero } & 9 & 13.44 & 2.651 & \\ \text { del Habla } & \text { Segundo } & 7 & 12.14 & 690 & \\ & \text { Tercero } & 7 & 12.71 & 1.254 & \\ & \text { Cuarto } & 10 & 12.70 & 2.541 & 4.946(.000)^{* *} \\ & \text { Quinto } & 5 & 12.20 & 2.490 & \\ & \text { Sexto } & 9 & 12.89 & 1.616 & \\ & \text { Séptimo } & 6 & 15.00 & 2.966 & \\ & \text { Octavo } & 4 & 18.75 & 1.500 & \\ & \text { Noveno } & 5 & 17.00 & 1.732 & \end{array}$




\begin{tabular}{|c|c|c|c|c|c|}
\hline Variable & Semestre & $\mathbf{N}$ & Media & D.T. & $F(p)$ \\
\hline Comunicación & primero & 9 & 16.11 & 1.965 & \\
\hline \multirow[t]{9}{*}{ Directa } & segundo & 7 & 13.57 & .976 & \\
\hline & tercero & 7 & 13.71 & 1.976 & $7.044(.000)$ ** \\
\hline & cuarto & 10 & 13.50 & 1.354 & \\
\hline & quinto & 5 & 12.80 & 2.387 & \\
\hline & sexto & 9 & 13.56 & 1.236 & \\
\hline & séptimo & 6 & 14.67 & 1.862 & \\
\hline & octavo & 4 & 18.00 & 1.633 & \\
\hline & noveno & 5 & 18.20 & 2.049 & \\
\hline & decimo & 8 & 12.25 & 2.866 & \\
\hline Estilos & primero & 9 & 29.56 & 2.698 & \\
\hline \multirow[t]{9}{*}{ Comunicativos } & segundo & 7 & 25.71 & .951 & \\
\hline & tercero & 7 & 26.43 & 2.370 & $8.007(.000) * *$ \\
\hline & cuarto & 10 & 26.20 & 3.225 & \\
\hline & quinto & 5 & 25.00 & 4.472 & \\
\hline & sexto & 9 & 26.44 & 2.404 & \\
\hline & séptimo & 6 & 29.67 & 4.367 & \\
\hline & octavo & 4 & 36.75 & 2.754 & \\
\hline & noveno & 5 & 35.20 & 3.271 & \\
\hline & decimo & 8 & 25.00 & 5.503 & \\
\hline
\end{tabular}

\subsection{Baremos}

En esta parte presentamos las escalas y los percentiles para determinar nivel de los estilos comunicativos y sus dimensiones, valido para los alumnos de Ciencias de la Comunicación de la UNCP.

Tabla 9: Escala para determinar niveles de los Estilos de Comunicación y sus dimensiones.

Estadísticos y Niveles Valoración del Habla Comunicación Directa Estilo de Comunicación

$\begin{array}{llll}\text { Media } & 13.59 & 14.37 & 27.96 \\ \text { D.T. } & 2.764 & 2.509 & 4.692 \\ \text { Puntaje Mínimo } & 8 & 9 & 19 \\ \text { Puntaje Máximo } & 20 & 20 & 40 \\ \text { Alto } & \text { De } 17 \text { a } 20 & \text { De } 17 \text { a } 20 & \text { De } 33 \text { a } 40 \\ \text { Medio } & \text { De } 10 \text { a } 16 & \text { De } 11 \text { a } 16 & \text { De } 23 \text { a } 32 \\ \text { Bajo } & \text { De } 8 \text { a } 9 & \text { De } 9 \text { a } 10 & \text { De } 19 \text { a } 22\end{array}$

\section{Escala}

La tabla nueve presenta tres niveles para la variable estilos comunicativos y sus dos dimensiones, considerando los respectivos puntajes directos para cada nivel.

\section{Percentiles}

La tabla 10 presenta los percentiles y sus puntuaciones directas correspondientes de los estilos comunicativos y de sus dimensiones. 
Tabla 10: Percentiles para los Estilos de Comunicación y sus dimensiones

$\begin{array}{llccc}\text { Estadísticos } & & \text { Valoración del Habla } & \text { Comunicación Directa Estilos Comunicativos } \\ \text { Mediana } & & 13.00 & 14.00 & 27.00 \\ \text { Rango } & 12 & 11 & 21 \\ \text { Mínimo } & 8 & 9 & 19 \\ \text { Máximo } & & 20 & 20 & 40 \\ \text { Percentiles } & 5 & 9.55 & 10.55 & 21.00 \\ & 10 & 10.00 & 12.00 & 23.00 \\ & 20 & 11.00 & 12.00 & 24.20 \\ & 30 & 12.00 & 13.00 & 25.30 \\ & 40 & 13.00 & 14.00 & 26.00 \\ & 50 & 13.00 & 14.00 & 27.00 \\ & 60 & 14.00 & 14.60 & 28.00 \\ & 70 & 14.00 & 15.00 & 29.70 \\ & 80 & 16.00 & 16.00 & 31.80 \\ & 90 & 18.00 & 18.00 & 36.80\end{array}$

\section{DISCUSIÓN}

Los resultados de la presente investigación demuestran las hipótesis planteadas, dando a conocer que el estilo de comunicación de los estudiantes de la UNCP se caracteriza por ser de puntuación alta.

Gudykunst, et al (1996) y Hall, (1978) señalan que la comunicación de Bajo Contexto involucra el uso de mensajes explícitos y directos en los cuales el significado está contenido principalmente en los mensajes transmitidos (este comunicador es abierto, auto-divulgador, los silencios significan que no hay nada más que decir, se es preciso), En ese sentido, los estudiantes de la UNCP, se caracterizan con este estilo comunicacional ya que prefieren comunicarse mucho y lo hacen sin ambages. Esto se demuestra cuando más de la mitad de los encuestados les gusta mucho hablar y compartir en situaciones sociales y lo hacen siempre y casi siempre. También Varona (2007), encontró que los trabajadores mexicanos emplean más la comunicación verbal y no verbal, como también la comunicación formal y no formal, y son más comunicativos que los trabajadores finlandeses y aluden más temas vinculados a la política o religión, en cambio los finlandeses más sobre deportes o recreación. Los empleados mexica- nos esperan recibir comentarios positivos y muestran mayor apertura comunicativa $y$, por ello, usan más los canales de comunicación, lo que se emparenta con los resultados de este estudio, puesto que se trata, en ambos casos, de sujetos latinoamericanos. Ahora bien, la investigación descubre que más de los dos tercios de estudiantes prefiere ir al grano cuando hablan con las personas. Esto se armoniza con la teoría propuesta por Miller, Nunnally y Wackman (1976), quienes describen entre sus cuatro estilos de comunicación verbal, la de tratar intencionalmente un tema de manera abierta y directa, estando en contacto con los pensamientos, sentimientos, intenciones propias, de la otra persona y de las implicaciones a la relación en forma honesta y responsable.

Sin embargo, el $53.8 \%$ de los encuestados prefiere siempre y casi siempre que los otros entiendan o deduzcan su opinión antes de tener que decirla directamente. Esta misma respuesta se corrobora con el $51.4 \%$ de estudiantes que, siempre y casi siempre, prefiere usar expresiones que matizan lo que dicen (por ejemplo" puede ser", " quizás"). Estas dos respuestas se enlazan con el estilo pasivo (no somos capaces de ex- 
presar abiertamente sentimientos, pensamientos y opiniones o lo hacemos con falta de confianza) propuesto por Van-der Hofstadt (2003).

Armenta y Diaz-Loving, (2008), recomienda que es necesario promover en las relaciones de pareja un estilo de comunicación positivo donde ambos miembros sean activos para lograr satisfacción en esta relación, además que cuando uno de ellos no se comunica de forma destructiva, fría, intolerante, severa, inexpresiva o distante, el otro es más platicador, oportuno y optimista al comunicarse, lo que produce una relación armoniosa. Esto se establece como un vínculo entre estas dos investigaciones, puesto que la recomendación anterior bien puede aplicarse a este trabajo.

Más de la mitad señala que, siempre y casi siempre, le presta más atención a lo que las personas dicen directamente, y no a lo que intentan decir o no dicen. En general, esta acumulación de respuestas manifiesta claramente que los estudiantes prefieren una comunicación más denotativa que connotativa. De igual forma, encontramos que los estudiantes son emocionales y defienden su posición cuando otras personas manifiestan una opinión contraria, o están en desacuerdo con ellas, manifestándolo abiertamente. Sobre el punto, más de la mitad de ellos informan que reaccionan de esta forma siempre y casi siempre.

Si partimos de los hallazgos de Flores (2010) que relaciona tipos de liderazgo con estilos de comunicación podríamos suponer que al estilo hallado de elevada valoración del habla y preferencia por la comunicación directa (comunicación agresiva) correspondería a un estilo de liderazgo autoritario, lo cual explicaría la facilidad de llevarlos a acciones de medidas de fuerza a través de expresiones breves y directas, ajenas a que puedan ser corroboradas en su veracidad.

En el otro lado, encontramos que un tercio de los encuestados no piensan que las personas que hablan poco son aburridas y poco interesantes, así como más de un tercio no le gustan las conversaciones superficiales, ni banales (hablar de la moda, futbol o televisión), mientras que menos de un tercio no le gusta hablar de lo mismo nunca o casi nunca. Quiere decir que, posiblemente por la edad, los jóvenes universitarios tienen entre sus temas preferidos de conversación la moda, el fútbol, los programas de televisión, pero también valoran las conversaciones serias, dada su condición de estudiantes universitarios.

En general, de la mano con Fensterheim y Baer (2003), podemos concluir que la comunicación entre los estudiantes de la carrera profesional de Ciencias de la Comunicación de la UNCP, pese a que es alta, tiende a ser pasiva (o no asertiva). Es aquel estilo de comunicación propio de personas que evitan mostrar sus sentimientos o pensamientos por temor a ser rechazados o incomprendidos o a ofender a otras personas. En exacta equivalencia, nuestro estudio determina que existen diferencias significativas en los estilos comunicativos de los alumnos de la UNCP según ciclo de estudios, con ligera tendencia a incrementarse según avanza el semestre académico de estudio.

Las diferencias significativas halladas de los estilos comunicativos según semestre y no en edades cronológicas, seria explicable por la influencia de la formación profesional que hace del estudiante comunicador con mayor valoración del habla y con énfasis en la comunicación directa. En cuanto al sexo, si bien las mujeres resultan con un mayor puntaje en los estilos de comunicación y sus dimensiones, estas diferencias resultan no significativas, aunque en la dimensión valoración del habla y en el total de estilos la significación se acerque a .005, esto merece corroborarse con estudios más amplios toda vez que Prior, et al (2011) si encontraron diferencias significativas.

Finalmente, se logró adaptar, validar y construir baremos para nuestro entorno del 
Cuestionario de Estilos de Comunicación de Gudykunst et al (1994, 1996). Los baremos, escalas y percentiles nos permiten diagnosticar los niveles de estilos comunicativos aplicables solo para los participantes del estudio o muestras similares. Estos hallazgos, ratifican la vigencia de la teoría, tal como también lo hicieron con otros instrumentos en México, Sánchez y Díaz (2003), Luna, Laca y Cedillo (2012). Otros diseñaron instrumentos originales como Uribe, García y Ramírez (2011) en México, Michaud y Warner (1997) en chile, y Flores (2010), Ludeña (2007) en el Perú

\section{REFERENCIAS}

- Armenta, C., \& Díaz-Loving, R. (2008). Comunicación y Satisfacción: Analizando la Interacción de Pareja. Psicología Iberoamericana, Vol. 16, núm. 1, 23 - 27.

- Chamoun-Nicolas, H. (S.F.) Los estilos de comunicación y el proceso de comunicación. Consultado el 07 de junio de 2013, de http://www.gestiopolis.com/ Canales4/mkt/esticom.htm

- Camargo U., Hederich M., C. (2007) El estilo de comunicación y su presencia en el aula de clase. Consultado el 07 de junio de 2013, de http://www.scielo.org. co/pdf/folios/n26/n26a01.pdf

- Fensterheim, H., Baer, J. (2003) No diga si cuando quiera decir no. México: Grijalbo.

- Flores, E. (2010). El tipo de liderazgo del director y los estilos de comunicación de los docentes, estudiantes y personal administrativo del Instituto Superior Pedagógico Público "Nuestra Señora de Lourdes". DRE-Ayacucho. Lima, Peru.

- Gudykunst, W., Ting-Toomey, S., Sudweeks, S. and Stewart, L (1994). Building Bridges: Interpersonal Skills for a Changing World. California: Allyn \& Bacon.

- Gudykunst, Matsumoto, Ting-Toomey, Nishida, Kim y Heyman (1996) The Influence of Cultural Individualism-Collectivism, Self Construal, and Individual Values on Communication Styles Across Cultures. Human Communication Research. Volume 22, Issue 4, pages 510-543.

- Hall, E.T. (1978). The silent language.New
York: Doubleday \& Company. Ludeña, L. (2007). Estilos de comunicación que establece la enfermera según opinión de los padres de niños quemados INSN. 2006. Tesis Licenciatura en Enfermería, UNMSM, Lima.

- Luna, A., Laca, F y Cedillo, L. (2012).Toma de decisiones, estilos de comunicación en el conflicto y comunicación familiar en adolescentes bachilleres. Enseñanza e investigación en psicología.Vol. 17, núm. 2: 295-311.

- Michaud y Warner (1997). Encuesta de estilos de comunicación. Santiago de Chile:Universidad de Chile.

- Miller, Nunnally y Wackman (1976). Psicología Organizacional en tipos de comportamiento, Washington, DF: Interpersonal Communication.

- Prior, M., Manzano, E., Villar, E., Caparrós, B., Juan, J. y Luz, E. (2011) Estilos comunicativos, vinculación universitaria y adaptación psicosocial.Revista de investigación educativa, 29 (2), 387 - 405.

- Sánchez, R. y Díaz, R. (2003) Patrones y estilos de comunicación de la pareja: Diseño de un inventario. Anales de psicología, Vol. 19, nº 2.

- Satir, Virginia (1986). Las cinco libertades. Virginia del Norte: Universidad de Virginia.

- Schmidt, V., Messoulam, N., Molina, M. y Abal, F (2008). Hacia una versión argentina de una Escala de Comunicación Padres-Adolescentes Revista Interamericana de Psicología, 42(1), 41-4

- Uribe, l., García, M. \& Ramírez, L. (2011). Relación entre estilos de comunicación y satisfacción sexual en estudiantes universitarios. Interamerican Journal of Psychology, Vol. 45, núm. 2, 157 -167.

- Van-der Hofstadt, C. (2003) El libro de las habilidades de comunicación.Madrid: Díaz de Santos.

- Varona, F. (2007). Diferencias en el estilo de comunicación entre empleados finlandeses y mexicanos cuando se comunican con sus jefes: un estudio comparativo. Signo y Pensamiento, XXVI (051), 137-149. 


\section{Curriculum vitae}

\section{Gaspar Orellana Méndez}

Psicólogo (1985)y magister en Psicología Educativa (1999) por la Universidad Nacional Mayor de San Marcos y con estudios de doctorado en Psicología en la Universidad Nacional Federico Villarreal (2002). Es docente ordinario en la categoría de principal en la Universidad Nacional del Centro del Perú y se ha desempeñado como profesor en pre y posgrado en diferentes universidades del país (UPLA, UCI, UNDAC y UNMSM). Fue Presidente de la Comisión de Admisión de la Universidad Peruana los Andes (1986), Director del Instituto de Investigación (1998) y Director de la Unidad de Posgrado (2001) en la Facultad de Educación, Decano de la Facultad de Ciencias de la Comunicación (2006) y Director de la Unidad de Posgrado (2013). Ha participado como ponente en eventos científicos nacionales e internacionales y ha publicado libros y artículos en psicología, educación y comunicación. Tiene trece asesorías de tesis de maestría y ha realizado una pasantía en la PUCP (2014). 
